\title{
El bluffware en la enseñanza de la ingeniería: síntomas y soluciones
}

\author{
D.F. Campos-Aranda \\ Facultad de Ingeniería de la UASLP. San Luis Potosí, SLP. \\ E-Mail: acampos@uaslp.mx \\ (recibido: septiembre de 1997; aceptado: febrero de 2001)
}

Resumen

"Desanima pensar en cuánta gente se escandaliza con la sinceridad, y a cuán poca escandaliza el engaño". Noel Coward.

\begin{abstract}
Inicialmente, este trabajo destaca el desarrollo vertiginoso que han tenido las ingenierías como consecuencia del apoyo computacional. En seguida, se explica en qué consiste la cultura de la apariencia (Bluffware), cuyo objetivo es simular una evolución académica en la institución. Se describen cinco causas de origen de tales actitudes y seis síntomas o consecuencias. También se proponen catorce medidas académicas correctivas, agrupadas según sus características en: medidas estructurales, medidas administrativas y medidas operativas. Finalmente, se plantean tres conclusiones relativas a las reflexiones críticas expuestas y dos comentarios de carácter personal al tópico expuesto.
\end{abstract}

Descriptores: enseñanza, cultura de la apariencia, educación, currículo, enfoque crítico.

\section{Abstract}

"It is discouraging to think how many people gets shocked about sincerity and how very few people gets shocked about deceit". Noel Coward.

The outstanding development of the various branches of engineering as a result of computer-related support is first emphasized. Then an explanation is made of the meaning of "bluffware" culture that has as an objective to give an impression of the academic development of an institution. Five sources of the origin of such attitudes are described together with six symptoms or consequences. Then 14 corrective academic measures are proposed, classified in terms of their characteristics as follows: 1) structural measures; 2) administrative measures; and 3) operative measures. Finally, three conclusions related to the critical reflections exposed are advanced, as well as two comments of personal nature on the topic discussed.

Keywords: teaching, bluffware, education, resume, critical focus.

\section{Marco de referencia}

Necesidad del cambio

Actualmente está de moda referirse a una necesidad apremiante de cambio en todos los órdenes, ello obedece primordialmente a la serie de eventos mundiales de carácter socio-político que han ocurrido en los últimos años; incluso, esta tendencia ha sido favorecida o agravada por los intensos comentarios de cambios climáticos y ambientales.

Lo paradójico es que la necesidad de un cambio en la educación técnica superior, debió manifestarse desde el inicio de la década de los años ochenta, o cuando menos, a mediados de ésta. Lo anterior, debido a que es en esta época cuando las diversas áreas o disciplinas de las ciencias y las tecnologías tuvieron un gran cambio 
en sus procedimientos de diseño y análisis, como resultado de lo accesible de los sistemas de cómputo personales (PC).

Para ilustrar lo anterior, debe mencionarse el vertiginoso desarrollo que han tenido recientemente la hidrología superficial, la geohidrología y los aprovechamientos hidráulicos; por ejemplo, en el campo de la modelación matemática determinística y probabilística, en los estudios de variabilidad espacial de parámetros y variables, y en los enfoques estocásticos y de optimización, entre otros. Estos desarrollos definitivamente no hubieran sido posibles sin la teorización de finales de los años sesenta y el apoyo computacional ya citado.

Por otra parte, resulta demasiado egoísta pensar que sólo estos campos o áreas de la ingeniería, hayan tenido un gran despegue, también ha ocurrido en todas las ciencias y sus disciplinas. Esto último, ha sido ignorado por comodidad, ya que de lo contrario, exigiría una actualización, tanto de los programas de las materias como de los apuntes y textos, pero sobre todo del personal docente y académico-administrativo.

\section{La definición propuesta para el Bluffware}

Como se sabe, a partir del advenimiento de las computadoras de alta velocidad en la década de los cincuenta, se definieron dos vertientes en este campo, éstas fueron el Hardware y el Software, el primero para englobar todos los avances en equipo de cómputo y el segundo para los desarrollos en programas y algoritmos. Lo lógico sería suponer que el fácil acceso a estas poderosas herramientas de cálculo, las PC, impulsarían a la enseñanza de las ingenierías en las escuelas y universidades de países en desarrollo, hacia su frontera de avance que se estaba generando en los países industrializados, pero no fue así, al contrario, se generó un retroceso que puede ser caracterizado por todas las acciones que se engloban en el Bluffware o cultura de la apariencia.

Con este término se intenta designar la apariencia de equipos y acciones que dan la impresión de un avance en el desarrollo de la enseñanza de las ingenierías; sin embargo, las han obstaculizado haciéndolas deficientes. Para ser más concretos, muchas instituciones han crecido en lo tangible (recursos humanos y organización, equipos de cómputo, edificios, laboratorios y gran número de eventos académicos), pero todo ello ien cuánto ha permitido mejorar la calidad académica y acrecentar el compromiso social de nuestros egresados?

La crisis de valores y sus consecuencias en el ámbito académico

Con fines prácticos se puede definir una crisis de valores como la ausencia total o parcial de la honradez, la lealtad, la responsabilidad y la ética; en resumen, la pérdida del honor. En general, se ha dejado de ser críticos, se ha abandonado el valor civil y se ha entendido erróneamente el término solidaridad. Aclaremos brevemente estas aseveraciones: es más cómodo y mucho más seguro para no meterse en problemas, el no criticar nada. El valor civil para denunciar lo que a nuestro juicio está mal, se ha sustituido por una solidaridad mal entendida con nuestros compañeros de trabajo y con la institución. Sobre este tema se puede desarrollar todo un ensayo; sin embargo, se considera mucho más importante citar cuáles han sido las consecuencias o síntomas de la crisis de valores que han originado este problema, entre otras causas, es importante mencionar la recesión económica, la cual ha invadido a la enseñanza superior, generando o produciendo el bluffware.

\section{Causas que han originado el bluffware}

1a. El atraso en el enfoque de la enseñanza de las ingenierías

Desde la mitad de la década de los años sesenta, Levi (1965) destacó la necesidad de cambiar de una enseñanza de la ingeniería informativa a una formativa, más básica, menos especializada y sobre todo mucho más moderna o actualizada con el desarrollo vertiginoso que ya estaba teniendo el diseño y análisis ingenieril. A mediados de los años ochenta se hicieron planteamientos concretos sobre la conveniencia de regresar a los programas de estudio con materias obligatorias únicamente, sin optativas, para buscar una formación integral sustentada en la consulta o investigación práctica para la comprensión y aplicación de las soluciones modernas que se estaban presentando en los diferentes problemas de la ingeniería. Tales planteamientos no se aceptaron y ahora los egresados no están capacitados para autoprepararse y ser competitivos contra los ingenieros que han acumulado experiencia profesional, como ha sido descrito por Campos (1993).

\section{2a. La mal entendida libertad de cátedra}

Como consecuencia del punto anterior, no se realizaron actualizaciones a los programas de estudio de cada materia, tampoco se revisó la seriación o continuidad del conocimiento, ni se evitó la duplicación de temas y "repasos", o más grave todavía, se marcó una ausencia de temas o tópicos básicos, de manera que aún los programas tienen severos errores y anomalías. ¿Por qué se favoreció esto? En parte por la mal entendida libertad de cátedra, la cual ha conducido a un escaso control o seguimiento de la enseñanza, del alcance de los temas impartidos y a la nula evaluación externa o ajena al 
profesor del conocimiento adquirido por los alumnos en cada materia. Todo esto se ha visto favorecido por la reducida normalización de la enseñanza, que se puede alcanzar a través de la adopción de un texto básico en cada cátedra y un estricto programa o lista de conceptos, procedimientos y actividades académicas de cada materia. Mientras no se "cuantifique y evalue la calidad de la enseñanza" estaremos dentro de la cultura del bluffware.

\section{3a. Escasa capacitación de los profesores}

Probablemente, lo accesible de los equipos de cómputo se adelantó a las necesidades de la enseñanza de las ingenierías, esto debido a la reducida participación del profesorado de ingeniería en cursos de posgrado y en talleres de actualización impartidos por los mismos académicos que cursaron estos grados durante las décadas de los años sesenta y setenta. Como consecuencia de lo anterior, no existía una necesidad real de apoyo de cómputo, dado que los procedimientos y técnicas de análisis y diseño en las ingenierías no habían evolucionado, la enseñanza seguía y, en algunas áreas, sigue siendo la misma de hace muchos años. Afortunadamente se crearon centros de cómputo y se impartieron este tipo de materias; sin embargo, no se generó software lo cual corrobora que se contribuyó al bluffware, ya que para casi cualquier asignatura se puede desarrollar bastante software de apoyo (Campos, 1996).

\section{4a. El papel real de los posgraduados}

Ante una política académica de escasa capacitación y transmisión del conocimiento entre profesores, ¿qué actitud tomaron y siguen adoptando los académicos posgraduados? Se han aislado y, en el mejor de los casos, se han dedicado a un desarrollo académico y profesional extraordinariamente personalizado, con reducidos o nulos beneficios para la enseñanza de las ingenierías y con aportaciones favorables al bluffware, por ejemplo, la institución puede reportar proyectos de investigación en desarrollo, contribuciones a congresos y otros foros, pero todo esto no ha mejorado, ni mejorarálos procesos de enseñanza de la ingeniería, al menos no sustancialmente.

\section{5a. Consecuencias de la investigación}

Actualmente se ha utilizado la investigación como parámetro de evaluación de las instituciones de educación superior, ésta ha servido para distanciar más a los profesores eminentemente docentes, de los que tie. nen horas dedicadas a actividades de investigación, pues éstos últimos publican en congresos internacionales, nacionales y otros foros, lo cual les otorga el derecho de asistir a tales eventos, no así, aquéllos que no publican. Pero además, los investigadores justifican su trabajo por medio de informes de proyecto, los cuales muchas veces son inaccesibles a los profesores hora-clase y sobre todo a los alumnos, debido a la sofisticada presentación teórica en que son revestidos, de lo contrario, dan la impresión de ser de mediana calidad.

\section{Sintomas principales del bluffarare}

\section{1o. Devaluación de las carreras universitarias}

En la actualidad, poseer un título universitario, sobre todo de nivel licenciatura, no garantiza tener o alcanzar inmediatamente un desarrollo profesional aceptable, tampoco asegura tener el acceso a un empleo medianamente bien remunerado. Aunque lo anterior tiene diversos orígenes, gran parte se debe a la lenta incorporación de los recién egresados al trabajo produc. tivo y a su baja competitividad contra los profesionales en activo.

\section{2o. Reducción de la matrícula}

Esta es la principal consecuencia del punto anterior, ya que ahora los jóvenes optan por soluciones más rápidas, o cuando menos, seleccionan las carreras de amplio espectro de posibilidades de desempeño, como las ingenierías civil y mecánico-eléctrica. Es entonces cuando las carreras de baja demanda o matrícula, reciben un paliativo, "aceptar alumnos rechazados de otras licenciaturas"; esto implica dos graves inconvenientes: a) son alumnos con nula vocación por la carrera en la que son incorporados y b) son los de más bajas calificaciones en los exámenes de selección. Desafortunadamente, esto también ocurre parcialmente en los posgrados.

\section{3o. Sostenimiento de carreras y posgrados}

Algunas carreras se siguen impartiendo con base en el número reducido de alumnos que optan por ellas, o que pertenecen a los que fueron rechazados en otras licenciaturas. Por otra parte, la matrícula en los posgrados obedece principalmente a la facilidad que da la institución para obtener el título de licenciatura con solo cursar un semestre en maestría o dos cuatrimes. tres en alguna especialidad; ciertos posgrados presentan escasa o nula inscripción por parte de los profesionistas en activo. 


\section{4o. Inercia al cambio}

Todas las causas citadas, así como los síntomas descritos, tienen efectos o consecuencias de mayor o menor cuantía ya sea en la inercia o aversión que se tiene al cuestionamiento, a la propuesta de soluciones, pero principalmente, a la escasa adopción de estrategias y actitudes para cambiar hacia una mejor formación a los alumnos y egresados de las ingenierías; la prueba contundente de ello es que no se han realizado tales cuestionamientos y cambios.

\section{5o. Hacia cero lectura}

Tanto los profesores como principalmente sus alumnos, tienden hacia el desinterés dedicado a la lectura técnica, ya sea en casa o en la biblioteca (Rugarcía, 1999). En este punto, la elaboración de informes de proyectos de investigación de carácter divulgativo y de material didáctico de presentación bilingüe, son soluciones que deben ser estimuladas para acelerar la lectura y compresión del idioma inglés.

6o. Falta de respeto a la institución y a los profesores

Es notorio como los alumnos no respetan las disposiciones de la institución y como atentan contra la conservación general de las instalaciones. Ahora el profesor de ninguna manera es visto con respeto y agradecimiento por su labor de cátedra. Además, en los pasillos de las instituciones de enseñanza superior ahora es común encontrar alumnos perdiendo el tiempo, o discutiendo de todo, menos de temas o tópicos de clase de ingeniería.

\section{Propuesta de acciones correctivas}

Las principales acciones que se visualizan son necesarias para abandonar la cultura del bluffware, han sido agrupadas bajo tres áreas conceptuales:

\section{Medidas correctivas académico-estructurales}

\section{1a. Reestructuración de carreras y posgrados}

Esto es, su actualización en alcances, forma de enseñarlas y su enfoque hacia las actuales y verdaderas necesidades de la sociedad moderna, tanto en sus aspectos teóricos, como técnicos y sociales. Lo anterior significa la actualización inmediata y expedita de los programas de estudio, la revisión de su funcionamiento y quizás el cambio a matrices curriculares de sólo materias obligatorias, además del establecimiento de programas de formación de docentes y la planeación a futuro de sus posgrados (Campos y Difurt 1992). 2a. Cierre definitivo o temporal de las carreras de escasa demanda o matrícula

Establecimiento de una mejor promoción en carreras que aún se consideren o visualicen importantes para la sociedad, o que sean consideradas básicas (de amplio espectro), o de futuro prometedor.

\section{3a. Creación de nuevas carreras o licenciaturas}

Esta creación debe ser en un contexto real que englobe las necesidades de la sociedad moderna, ya sea urbana o rural y haciendo uso de los recursos humanos de toda la institución; por ejemplo, Campos (1994) ha propuesto la creación de la licenciatura en ingeniería ambiental, de carácter multidisciplinario para las facultades de Ingeniería, Química, Medicina y Derecho.

\section{Medidas correctivas académico-administrativas}

4a. Mayor participación académica y administrativa del personal docente posgraduado

Ello implicará un punto de vista más amplio y actual de cualquier área o campo de la ingeniería, tanto en la cátedra como en las actividades directivas de nivel licenciatura, no sólo de los posgrados. Esto implica, que si un profesor se posgraduó en análisis estructural, su carga docente debe quedar comprendida en tales materias, sin importar a quién o quiénes se lesione académica o administrativamente.

\section{5a. Se debe normalizar o estandarizar la enseñanza}

Al disponer de programas actuales, detallados y ambiciosos (en teoría y en aspectos prácticos) del contenido de cada materia, será posible estandarizar la enseñanza y mejorar notoriamente su control, ya que en la actualidad es prácticamente nulo. De esta forma, la enseñanza se realizará con base en un texto previamente seleccionado, si existe, o integrado (ensamble de notas) si no lo dispone, de tal manera que la docencia se estandarice y se logren efectuar exámenes generales si se tienen varios grupos de una materia. Estos exámenes se integrarán con base en los catálogos de problemas de cada asignatura (libro de problemas).

6a. Se debe eliminar la permanencia en puestos directivos

Esta eliminación, debe realizarse con carácter eminentemente académico, por períodos mayores a su designación, pues actualmente, todo cambio será benéfico para las 
áreas, carreras e institución en general, ya que ayudará a "romper" la inercia del cambio.

7a. Se debe buscar una transparencia total en el otorgamiento de los premios al desempeño académico

Ya que cada año la evaluación de los profesores se ha ido oscureciendo, por lo tanto, se ha hecho menos objetiva, llegando al extremo de una asignación secreta del nivel alcanzado y del monto por recibir.

\section{Medidas correctivas académico-operativas}

\section{8a. Control efectivo de la docencia}

Se debe ejercer un verdadero control cuando menos mensual, acerca del avance académico en cada asignatura, y al final del semestre evaluar la cátedra con base en la revisión de notas tomadas por los alumnos, tareas realizadas y apuntes (fotocopias) utilizados por el profesor y entregados a los alumnos; en resumen, todo el material de apoyo deberá ser examinado por la academia de área respectiva para emitir un dictamen sobre el desempeño del profesor en tal asignatura.

\section{9a. Ampliar el semestre escolar}

Para cumplir con la mayor carga académica y alcanzar un mejor proceso enseñanza-aprendizaje, es necesario disponer de más tiempo. Se propone que el semestre incluya 20 semanas efectivas de clases, tres para exámenes finales y extraordinarios, dos semanas de vacaciones y una de planeación académica y organización de cursos del futuro trabajo semestral.

10a. Eliminación de los exámenes a título de suficiencia

Para alcanzar una mejor eficiencia académica, se considera necesario eliminar tal opción de evaluación y se sugiere permitir cursar la materia hasta tres veces. Es una falacia pretender evaluar todo el conocimiento impartido sobre una materia, a través de un sólo examen escrito u oral.

\section{1a. Compactación de horarios}

Con la idea de optimizar el uso del tiempo por parte de alumnos y profesores, se deben instituir los horarios compactos, en donde los alumnos acudan únicamente por las mañanas durante los dos primeros años y por las tardes a partir del tercer año. Los estudiantes de tiempo completo podrán dedicar toda la mañana a sus tareas, lecturas e investigaciones, y los de medio tiempo, podrán comenzar a desempeñarse profesionalmente, pero sin reducir su rendimiento académico de tiempo parcial.

\section{2a. Implementación de conferencias de expertos}

Es necesario impartir cuando menos una conferencia en cada cátedra, orientada a destacar el nivel elemental, pero básico de los conocimientos del alumno, así también, mostrar la frontera del avance en esa materia. Esto permitirá, que los alumnos y profesores, "abran los ojos" hacia el amplísimo mundo de la ingeniería, en sus acepciones como ciencia y tecnología.

13a. Mayor exigencia de nivel académico o técnico durante los procesos y opciones de titulación

Actualmente, se tienen varias opciones de titulación, que van desde realizar un trabajo recepcional, cursar un semetre en un posgrado, o bien, realizar un curso de actualización con duración superior a 165 horas. En general, se ha olvidado que ésta es la etapa final de formación del futuro profesionista, no un mero trámite administrativo; claro que esto es más cómodo, ya que eleva la eficiencia terminal y además genera recursos económicos para la institución.

\section{4a. Formación de comités académicos y de} academias

Esto es crucial por área o campo del conocimiento dentro de cada licenciatura y posgrado, para iniciar de inmediato la 1a., 5a., 8a. y 11a. de las acciones correctivas propuestas, esto es, la reestructuración de carreras y posgrados, la normalización de la enseñanza y el mayor control en el nivel técnico de los trabajos u opciones de titulación.

\section{Conclusiones}

Primera: debido a la falta de una verdadera planeación de las actividades académicas orientadas hacia metas reales (falta de un proyecto educativo), ciertas instituciones de educación superior han adoptado acciones propias de una cultura de la apariencia (bluffware), esto es, dar la impresión de un avance académico, cuyas consecuencias negativas se detectan en la calidad científico-técnica y de compromiso social y ético de sus egresados.

Segunda: casi todas las instituciones de educación superior han crecido en lo tangible, por ejemplo, en recursos humanos, equipos de cómputo, instalaciones (laboratorios, salones, auditorio, etc.) y eventos académicos, pero han descuidado la esencia del proceso enseñanza-aprendizaje 
que es precisamente su evaluación, verificación y corrección, lo cual es más necesario.

Tercera: la propuesta de estas catorce medidas correctivas en este trabajo, implican muchos cambios y grandes esfuerzos para su implementación, pero únicamente bajo tales estrategias se podrá superar la cultura de la apariencia y dirigirse hacia la verdadera calidad en la docencia, lo cual implica la superación académica que a todos beneficia.

\section{Comentarios finales personales}

Primero: como consecuencia de mi desempeño académico dentro de una institución pública de enseñanza de las ingenierías, las observaciones y comentarios citados se refieren exclusivamente a la enseñanza de la ingeniería civil a nivel licenciatura y de sus posgrados en el área de hidráulica; sin embargo, considero que fácilmente pueden ser extrapoladas estas ideas y análisis críticos a otras instituciones en sus licenciaturas y posgrados; asímismo, servir de 'alarma' para no incurrir en tales deficiencias.

Segundo: lógicamente los análisis críticos que han sido expuestos, obedecen en primer término a un sentimiento de culpa por la escasa contribución que mi trabajo como docente e investigador ha tenido en la preparación académica de los alumnos de ingeniería civil y de otras carreras afines.

\section{Referencias}

Campos-Aranda D.F. (1993). Enseñar a prepararse. Ingeniería Civil, No. 294, octubre, 14-19. CICM.

Campos-Aranda D.F. (1994). Propuesta para el establecimiento de una licenciatura en ingeniería ambiental en la UASLP. III Coloquio Internacional: Curriculum y Siglo XXI. Ciudad Universitaria, UNAM. Junio. México, DF.

Campos-Aranda D.F. (1996). Software Development at the Agroclimatology Program of the Irrigation and Drainage Postgraduate Courses at the UASLP. 6th International Conference on Computers in Agriculture, Theme: Weather, Cancún, México, junio.

Campos-Aranda D.F. (1997). Sobre las acreditaciones en ingeniería civil: evaluación académica de los programas de enseñanza, exámen de calidad profesional y certificación de profesionales en activo. Ingeniería, Vol. LVII, No.4, octubre-diciembre, 279-285.

Campos-Aranda D.F. y Difurt-Candelaria A. (1992). A Novel Program for the Master's Degree in Hydraulic Engineering in the Universidad Autónoma de San Luis Potosí. Conference and Workshops in Water Resources and Environment: Education, Training and Research. Session VI-A, Colorado State University, Fort Collins, CO., USA, julio.

Levi L.E. (1965). Teorías y métodos de las matemáticas aplicadas. Universidad Nacional Autónoma de México. Segunda Edición (1980). México.

Rugarcía T.A. (1999). ¿Por qué no leen los estudiantes? Ciencia y Desarrollo, Vol. XXV, No. 146, 46-53.

Daniel Francisco Campos-Aranda. Concluyó la licenciatura de ingeniería civil en 1972 en la Escuela de Ingeniería de la UASLP. La División de Estudios de Posgrado de la Facultad de Ingeniería, UNAM le otorgó los títulos de maestro (1980) y doctor (1987) en ingeniería hidráulica y aprovechamientos hidráulicos, respectivamente. En 1989, obtuvo la medalla "Gabino Barreda" de la UNAM. Ha publicado en revistas nacionales como: Ingeniería hidráulica en México y Agrociencia. Asimismo, ha participado en congresos nacionales e internacionales. Actualmente es investigador nacional y profesor de la División de Educación Continua en la Facultad de Ingeniería, UNAM. 